\title{
Liderazgo entre iguales en equipos deportivos: una revisión camino a la integración
}

\section{An Integrated Vision of Peer Leadership in Sports Teams: A Review}

\author{
Julio Torrado Quintela \\ Universidade Lusófona do Porto, Portugal
}

Disponible online 31 de agosto de 2012

\begin{abstract}
El estudio del liderazgo deportivo se ha centrado de forma mayoritaria en la figura del entrenador, dando menor importancia al liderazgo ejercido por algunos jugadores entre sus compañeros. Sin embargo el desarrollo diario en el ámbito aplicado nos muestra continuamente la importancia del jugador líder dentro de un equipo deportivo, dada la influencia que tiene sobre los demás jugadores y su repercusión en todos los procesos grupales. Este trabajo ofrece una revisión teórica acerca de las investigaciones realizadas, centrándose en los avances producidos en el estudio del liderazgo informal desarrollado por un miembro del equipo entre sus iguales, tanto en los aspectos conceptuales como en los sistemas de evaluación del liderazgo que se han desarrollado. De manera particular se pretende ofrecer una visión integradora tanto a nivel teórico como metodológico a través de las publicaciones más recientes, que permita una establecer un punto de referencia actual y señale posibles líneas futuras de investigación.
\end{abstract}

Palabras clave: Liderazgo; Informal; Deporte; Iguales; Evaluación.

In general, studies on sports leadership have focused on the coach, paying less attention to the leadership shown by some athletes in relation to their peers. However, their daily practice shows us the importance in sports teams of peer leaders, due to their influence on the other players and their impact on all the group processes. This study provides a theoretical overview of the literature, focusing on advances in the study of peer leadership in relation to the conceptual issues and the assessment systems used. Specifically, it offers an integrated vision, at the theoretical and methodological level, based on recent publications, which allows us to establish a current point of reference and suggests future lines of research.

Key words: Leadership; Informal; Sport; Peer; Evaluation.

Correspondencia: Faculdade de Psicologia. Universidade Lusófona do Porto. Rúa Augusto Rosa, 24, 4000-098 Porto. Portugal. Tlf: 0035122 2073230 / 626703527 (tlf móvil España) // Fax: 0035122 2073237. E-mail: julio.torrado@ulp.pt 
El liderazgo es un proceso grupal por el cual un individuo ejerce una influencia sobre los demás miembros del grupo, que contribuye al alcance de los objetivos comunes. Todas las personas, al interactuar, se influyen mutuamente unas a otras, sin embargo existen factores que determinan que algunas tienen la capacidad de influir en las demás en mayor medida en que las demás lo harán en ellas (Homans, 1961). Esta influencia, además, repercute positivamente en procesos grupales como la cohesión, la motivación o la comunicación.

En cuanto al estudio del liderazgo, es necesario establecer la diferencia entre los grupos grandes y los grupos pequeños. En los primeros (grandes sociedades, estados, empresas, movimientos sociales...), se ha determinado la especial relevancia de aspectos como el carisma (Bass, 1985; Jacquart y Antonakis, 2009). En cuanto a los segundos, se entiende por grupos pequeños aquellos en los que sus miembros interactúan y establecen relaciones personales, las cuales toman precisamente un importante peso en este proceso (Zaccaro, Rittman y Marks, 2001). Dentro de los grupos pequeños es necesario afrontar la dicotomía planteada entre el liderazgo formal y el informal. Se entiende por liderazgo formal aquel que viene definido por la estructura formal del grupo. Su autoridad sobre el grupo está basada en el concepto de poder, asociado al puesto jerárquico de la estructura que ocupe el sujeto que ejerza tal liderazgo y no al individuo en sí mismo (Peus, Weisweiler y Frey, 2009). Además de éste, otro tipo de liderazgo puede convivir en un grupo: el liderazgo informal (Bednarek, Benson y Mustafa, 1976). Este liderazgo está fundamentado en el concepto de influencia, no estando determinado por la estructura previa del grupo (Wheelan y Johnston, 1996), y su función puede terminar siendo desempeñada por cualquier miembro del grupo sea cual sea su status inicial en éste (Northouse, 2007).

Un contexto que permite observar y estudiar la conducta grupal de manera privilegiada es el ámbito deportivo (LeUnes, 2008). Dentro de los equipos deportivos, además, se presenta de manera clara la referida dicotomía entre el liderazgo formal e informal. La investigación de este proceso en el ámbito del deporte ha estado centrada, de forma mayoritaria, en el estudio del liderazgo formal del entrenador. Sin embargo, existe un liderazgo informal que ejercen algunos jugadores entre sus iguales, los demás miembros del equipos deportivos (Carron, Hausenblas y Eys, 2005; Hernández-Mendo y Canto, 2003). Es sobre esta figura de líder informal sobre la que se centra fundamentalmente esta revisión, en la que veremos las investigaciones más importantes realizadas en la definición de un marco teórico y los métodos utilizados para la evaluación de dicho liderazgo.

\section{Modelo teórico}

Si tomamos como punto de partida el liderazgo formal representado por el entrenador, la referencia principal se establece en el Modelo Multidimensional del Liderazgo (Chelladurai, 1978, 1990; Chelladurai y Saleh, 1978). Este modelo plantea la existencia de antecedentes, que son la conjunción de diversas características (personales del líder, situacionales y de los miembros del grupo), que determinan la conducta (que es a su vez una combinación de la conducta requerida, la preferida y la conducta que realmente se lleva a cabo). Tras esta conducta existen unas consecuencias que retroalimentan el proceso que define el modelo.

Aunque es cierto que existen semejanzas entre el liderazgo que desarrollan los entrenadores de equipos deportivos y el ejercido por un jugador entre sus iguales, ambos tipos de liderazgos no pueden ser entendidos y analizados en los mismos términos, ya que la forma de ejercerlo sobre el grupo y la manera de percibirlo por parte de los miembros del equipo es diferente (Jowett, 2008). En un estudio comparativo los deportistas señalaron que los entrenadores tienden a centrarse en conductas destinadas a la mejora del nivel de juego, además de mantener un perfil más autoritario. Por el contrario, los jugadores que ejercen de líderes muestran un perfil más democrático, mayor apoyo social y ofrecen más feedback positivo (Loughead y Hardy, 2005).

En la definición de un marco teórico común del liderazgo informal, inicialmente se adoptó la tradición clásica sobre el liderazgo que plantea una figura compuesta por dos dimensiones conceptuales, una de carácter instrumental y otra de carácter social. La dimensión instrumental, también llamada orientación a la tarea, engloba las conductas centradas en el cumplimiento de objetivos, la obtención de resultados, el desarrollo de los procedimientos o el reparto de funciones. La dimensión social hace referencia a las conductas orientadas a mantener un buen clima y una alta satisfacción entre los compañeros, fortalecer la cohesión y promover unas buenas relaciones personales entre los miembros del equipo.

Si bien el planteamiento teórico en contextos no deportivos se basó inicialmente en el concepto de diferenciación de rol, que entendía que el líder se definía por su tendencia a priorizar conductas de una orientación sobre la otra (dando lugar a líderes orientados socialmente o líderes orientados a la tarea), la investigación específica en el campo deportivo mostró que los líderes de grupos tienden a puntuar alto en ambas dimensiones y no preferentemente en una más que en otra, lo que se ha venido a denominar como integración de rol. Rees (1983) llevó a cabo un estudio en el ámbito del baloncesto en el que se evaluaron la orientación a la tarea (la consecución de objetivos) y la orientación social (la cohesión del grupo), evaluando la importancia de ambas en la formulación de un modelo teórico de liderazgo, concluyendo la existencia de dicha integración de rol. Los líderes puntúan alto en ambas dimensiones y no necesariamente se decantan por alguna de ellas como estilo de liderazgo. Posteriormente Rees y Segal (1984), tomando como muestra equipos de fútbol, pusieron a prueba la propuesta sobre la integración de rol, obteniendo resultados que reafirmaban los anteriores. Otros autores han tratado de contribuir a esta discusión con investigaciones similares, que ofrecen resultados que apoyan el concepto de la integración de rol (Kozub y Pease, 2001), aunque en ciertos casos se ha señalado que los deportistas masculinos valoran de modo ligeramente superior a los 
líderes que son percibidos como más orientados a la tarea (Todd y Kent, 2004). En la misma línea de la integración de rol también se ha comprobado que los grupos con líderes que puntúan alto en ambas dimensiones muestran una mayor perspectiva de resultados (Kim, 1992).

Algunos autores han desarrollado una idea que asume estas dos dimensiones de tarea y social (que engloban bajo la etiqueta de "acciones internas"), e implica además una tercera dimensión en la concepción del jugador líder, la "acción externa", que haría referencia a la relación con el entorno y la imagen externa que el líder del equipo ha de transmitir (Kogler-Hill, 2001). En función de esta interpretación el líder ejerce como portavoz, imagen y representación del equipo. Algunas investigaciones recientes al respecto parecen respaldar esta idea (Dupuis, Bloom y Loughead, 2006; Eys, Loughead y Hardy, 2007; Loughead, Hardy y Eys, 2006) que, sin embargo, tiene su origen en trabajos de hace ya algunas décadas (Mosher, 1979). En todo caso, esta dimensión parece más relacionada con equipos de alto rendimiento que puedan demandar esa necesidad, y donde la función de representación del líder tenga un sentido claro. En otras situaciones no parece tan evidente que sea una dimensión a tener en cuenta, ya que parece más propiciada por determinados entornos (como el de los equipos de alto rendimiento) que por el propio grupo en sí.

Con el objetivo de profundizar en la construcción de un marco teórico, se han desarrollado diversos estudios focalizados en alguna de las dimensiones. Algunos autores investigaron sobre la relación existente entre las variables de liderazgo y la afinidad personal dentro del grupo, mostrando la tendencia de los jugadores a considerar al líder como alguien muy cercano en lo personal y concederle un alto estatus en las relaciones de amistad (Weiss y Stuntz, 2004; Yukelson, Weinberg, Richardson y Jackson, 1983). Moran y Weiss (2006) analizaron la relación entre el género y la orientación social del jugador líder, teniendo ésta más peso todavía en equipos femeninos que masculinos. Esta última idea también ha sido apuntada en el análisis de la relación entre liderazgo y cohesión en grupos de mujeres que realizan actividad física conjuntamente (Caperchione, Mummery y Duncan, 2011). Wright y Côtè (2003) realizaron una investigación con equipos deportivos universitarios en Canadá en la que determinaron la importancia fundamental de la empatía y de unos fuertes valores éticos. El concepto de empatía también ha sido señalado en posteriores trabajos como un aspecto básico en la definición de un modelo de liderazgo (Arce, Torrado, Andrade, Garrido y de Francisco, 2008).

En el aspecto referido a la orientación a la tarea se ha encontrado que, en términos generales, existe una correlación positiva entre el nivel de juego de los deportistas y su capacidad de liderazgo del equipo (Gill y Perry, 1984; Glenn y Horn, 1993; Wright y Côtè, 2003; Yukelson et al., 1983). Parece existir una correlación positiva todavía más intensa entre la ubicación en el campo y esa capacidad de liderazgo, mostrando que los jugadores que son señalados como líderes tienden a ocupar las posiciones más influyentes en el juego. Un primer estudio sobre este tipo de relación, llevado a cabo en baloncesto, ya establece la hipótesis de que los jugadores con una mayor capacidad de liderazgo son, potencialmente, los más ideales para ejercer como directores del juego del equipo (Grusky, 1963). En el mismo sentido el trabajo de Gill y Perry (1984) señala la posición en el campo como una importante variable en relación a la capacidad de liderazgo en un estudio en equipos escolares. También Klonsky (1991) apoya esta idea, y además señala que este tipo de relación se aprecia de manera especial en el béisbol, deporte en el que los líderes casi pueden establecerse según la posición que ocupen en el campo siendo los más probables aquellos que están más en contacto con las situaciones decisivas. Sobre esta misma temática de la ubicación en el campo de juego, está planteada también una hipótesis recíproca a la anterior, planteando que es la ubicación en posiciones centrales e influyentes en el deporte del fútbol la que favorece que éstos jugadores se conviertan en líderes de los equipos (Lee, Coburn y Partridge, 1983). El estudio de esta relación entre la posición de los jugadores en el planteamiento del juego y la capacidad de liderazgo propicia que podamos establecer la existencia de una relación entre ambas características, pero que no sea posible establecer una relación causal ni en una dirección ni en la otra debido a los datos contrapuestos hallados sobre el tema.

Una reciente línea de investigación ha tratado de aportar una visión integradora del punto de vista clásico bidimensional (social y de tarea) con los trabajos destinados a profundizar en cada una de las dos grandes dimensiones. Para ello, se parte de un modelo simple de dos grandes dimensiones conceptúales, orientación social y orientación a la tarea. Las investigaciones que se han centrado en conocer las variables que, siendo específicamente de corte social, están más relacionadas con la capacidad de liderazgo, sugiere la necesidad de delimitar con mayor precisión la composición de tal orientación. Por ello se ha apuntado la existencia de cuatro dimensiones conceptuales de segundo nivel (ver figura 1) referentes a dicha categoría social, basadas en los conceptos que se han revelado como más importantes históricamente y que contribuyen a una mejor definición de este aspecto y, por tanto, también del modelo en conjunto (Arce, Torrado, Andrade y Alzate, 2011; Torrado, 2009):

- Apoyo Social: hace referencia a las muestras de ayuda y ánimo tanto en situaciones positivas como negativas (Chelladurai, 1978; Loughead y Hardy, 2005).

- Influencia en la toma de decisiones: otorga al líder un papel fundamental en las decisiones grupales e individuales a través del concepto de influencia (Bass, 1985; Homans, 1961)

- Valores deportivos: relaciona la representatividad del líder en cualidades intangibles positivas, tales como honestidad, honradez y responsabilidad (Wright y Côtè, 2003).

- Empatía: define la capacidad de la identificación emocional del líder con los demás miembros del grupo (Arce et al., 2008; Weiss y Stuntz, 2004, Wright y Côtè, 2003; Yukelson et al., 1983). 
Figura 1

Modelo teórico propuesto por Arce, Torrado, Andrade y Alzate (2011)

\begin{tabular}{|c|c|c|c|c|}
\hline \multicolumn{5}{|c|}{ Liderazgo Deportivo } \\
\hline \multicolumn{4}{|c|}{ Orientación Social } & \multirow{2}{*}{$\begin{array}{l}\text { Orientación a la Tarea } \\
\text { Orientación a la Tarea }\end{array}$} \\
\hline Empatía & $\begin{array}{c}\text { Influencia en la Toma de } \\
\text { Decisiones }\end{array}$ & Valores Deportivos & Apoyo Social & \\
\hline $\begin{array}{l}\text { Se identifica con los } \\
\text { sentimientos de los } \\
\text { compañeros }\end{array}$ & $\begin{array}{l}\text { Su punto de vista es } \\
\text { importante a la hora de } \\
\text { tomar decisiones entre los } \\
\text { compañeros }\end{array}$ & $\begin{array}{l}\text { Se comporta de forma } \\
\text { honesta }\end{array}$ & $\begin{array}{l}\text { Da señales de satisfacción } \\
\text { cuando los compañeros lo hacen } \\
\text { bien }\end{array}$ & Lo da todo en los partidos \\
\hline $\begin{array}{l}\text { Siente lo que le pasa a los } \\
\text { compañeros }\end{array}$ & $\begin{array}{l}\text { Los compañeros tienen en } \\
\text { cuenta su opinión }\end{array}$ & Actúa de manera razonable & $\begin{array}{l}\text { Aprovecha los buenos momentos } \\
\text { para dar confianza a los } \\
\text { compañeros }\end{array}$ & Se esfuerza en los partidos \\
\hline $\begin{array}{l}\text { Comprende los problemas } \\
\text { de los compañeros }\end{array}$ & $\begin{array}{l}\text { Su opinión ejerce } \\
\text { influencia sobre los } \\
\text { compañeros }\end{array}$ & $\begin{array}{l}\text { Muestra un } \\
\text { comportamiento maduro }\end{array}$ & $\begin{array}{l}\text { Felicita a los compañeros cuando } \\
\text { lo hacen bien }\end{array}$ & Se emplea a fondo en los partidos \\
\hline $\begin{array}{l}\text { Comprende el sentir de los } \\
\text { compañeros }\end{array}$ & $\begin{array}{l}\text { Tiene mucho peso en las } \\
\text { decisiones entre } \\
\text { compañeros }\end{array}$ & $\begin{array}{l}\text { Hace las cosas con } \\
\text { honradez }\end{array}$ & $\begin{array}{l}\text { Muestra entusiasmo cuando se } \\
\text { están haciendo las cosas bien }\end{array}$ & Entrena duro \\
\hline $\begin{array}{l}\text { Percibe los problemas de } \\
\text { los compañeros }\end{array}$ & $\begin{array}{l}\text { Su opinión es respetada por } \\
\text { los compañeros }\end{array}$ & Actúa de manera reflexiva & $\begin{array}{l}\text { Defiende a los compañeros en } \\
\text { situaciones difíciles }\end{array}$ & Se prepara a conciencia para competir \\
\hline $\begin{array}{l}\text { Comparte los sentimientos } \\
\text { de los compañeros }\end{array}$ & $\begin{array}{l}\text { Se punto de vista convence } \\
\text { a sus compañeros }\end{array}$ & $\begin{array}{l}\text { Se comporta de manera } \\
\text { responsable }\end{array}$ & $\begin{array}{l}\text { Apoya a los compañeros en los } \\
\text { momentos malos }\end{array}$ & Se toma los entrenamientos en serio \\
\hline
\end{tabular}

Las aportaciones teóricas parecen confluir, de forma general, en la concepción de un modelo bidimensional del liderazgo, social y tarea, y los últimos trabajos pretenden integrar las investigaciones precedentes en un modelo que recoja de manera clara este planteamiento con la atención específica a la dimensión social y las variables que la componen. Sin embargo se antoja necesario someter esta última propuesta a una mayor discusión con el objetivo de obtener un modelo que pueda servir de marco de referencia consistente para el futuro. Además de consolidar un modelo bifactorial, las investigaciones futuras debieran profundizar en las dimensiones conceptuales de segundo nivel para comprobar la estructura de la dimensión social, así como para analizar posibles subdimensiones de la orientación a la tarea.

\section{Metodología de evaluación}

En este punto si, al igual que en el apartado teórico, tomamos como punto de inicio el liderazgo formal representado por el entrenador, encontramos también la referencia principal en los trabajos iniciales de Chelladurai (1978), que progresaron hasta la propuesta de la Leadership Scale for Sports (LSS, Chelladurai y Saleh 1980). La LSS ha sido traducida, validada y/o adaptada a diversos contextos culturales e idiomas como el portugués (Serpa, Lacoste, Antunes, Pataco y Santos, 1988), español (Crespo, Balaguer y Atienza, 1994), sueco (Isberg y Chelladurai, 1990), francés (Lacoste y Laurencelles, 1989), finlandés (Liukkonen y Salminen, 1989) o japonés (Chelladurai, Imamura, Yamaguchi, Oinuma y Miyauchi, 1988).
La LSS está diseñada para aplicarse en equipos deportivos con el objetivo de medir la capacidad de liderazgo del entrenador. Al jugador se le administra en dos ocasiones y se le pide, en la primera vez, que evalúe cómo sería su entrenador ideal; en la segunda se le pide que evalúe a su entrenador actual. Además, por otro lado, el entrenador también se auto-evalúa acerca de su comportamiento. Los resultados son comparados entre sí. La escala consta de 40 ítems que se agrupan en 5 grandes dimensiones:

- Entrenamiento e instrucción: referido a cómo el entrenador intenta que sus jugadores mejoren sus habilidades, cómo transmite instrucciones y decisiones técnicas, etc.

- Comportamiento democrático: referido al comportamiento del entrenador orientado a que los jugadores participen en las decisiones sobre el grupo y tengan libertad para expresarse y participar de las cuestiones grupales.

- Comportamiento autocrático: referido al comportamiento del entrenador basado en conductas más autoritarias y dando menos libertad al jugador.

- Apoyo Social: referido a una actitud propensa a mejorar el clima grupal, la cohesión y los aspectos sociales del equipo. - Feedback positivo: referido a la capacidad del entrenador de gestionar los refuerzos y los castigos hacia los jugadores.

La escala LSS ha sido revisada y modificada en investigaciones posteriores (Zhang, Jensen y Mann, 1997), si bien es la escala original la que se mantiene como principal referencia. Sin embargo parece natural asumir que si desde un punto de 
vista teórico el liderazgo formal e informal presentan diferencias entre sí, será necesario que sean evaluados de manera diferente y, por tanto, será preciso desarrollar métodos de evaluación específicos para el liderazgo informal.

Las primeras investigaciones sobre el jugador líder establecieron como método para señalar al líder la atribución directa de tal capacidad. Tropp y Landers (1979) pidieron a los jugadores de un equipo que puntuaran en la característica de "liderazgo" a cada uno de sus compañeros. Gill y Perry (1984) utilizaron el mismo sistema e incorporaron, además, la tarea de ordenar a los compañeros en función de su "influencia de liderazgo", estableciendo una media de ambas puntuaciones para obtener un valor final.

Con la intención de dotar de mayor profundidad y matiz a la evaluación de este tipo de liderazgo, se utilizaron instrumentos de medición más completos. En un estudio comparativo entre el liderazgo del entrenador y del jugador, Loughead y Hardy (2005) utilizaron el LSS, con ligeras alteraciones, para evaluar también el liderazgo que ejercen algunos jugadores. También Holmes, McNeil, Adorna y Procaccino (2008) utilizaron esta misma escala, aunque en este caso en la versión revisada, para un estudio en el que se pretendía diferencia entre líderes "dentro del campo" y líderes "fuera del campo", intercambiando el término "coach" de la escala original por el término "peer leader". Sin embargo, en ninguno de los casos se ofrece información sobre el hecho de que la escala, y sus alteraciones, hubieran sido validadas previamente para tal fin. Kim (1992) analizó la relación entre el nivel de ejecución del equipo y los posibles estilos de liderazgo (social y/o de tarea) que presentaban jugadores de distintos equipos escolares japoneses. Para ello utilizó una escala de liderazgo creada originalmente en el ámbito organizacional y adaptada posteriormente para el contexto deportivo (Banzai, 1989), aunque se le puede achacar el mismo problema de no informar de su validación.

Otro de los métodos empleados ha sido la evaluación cualitativa. Dupuis, Bloom y Loughead (2006) analizaron las conductas valoradas como más importantes para el ejercicio del liderazgo, realizando entrevistas a capitanes de equipos deportivos de institutos y preguntándoles acerca de las características que creían que más influían en su relación con el grupo.

En algunos casos también se han utilizado métodos como el sociograma y las comparaciones por pares para evaluar la capacidad de liderazgo de los distintos miembros de un equipo (Chen, 2006), si bien esta metodología no ha tenido todavía un elevado impacto en el estudio del liderazgo deportivo, siendo predominante la utilización de la metodología selectiva.

Por último existe una serie de investigaciones en las que se agrupan aquellos trabajos que han tratado de construir y desarrollar instrumentos específicos para la evaluación del liderazgo ejercido por ciertos jugadores. Glenn y Horn (1993) desarrollaron el Sport Leadership Behavior Inventory (SLBI), en una investigación con equipos de fútbol femenino escolar en EE.UU., con el objetivo de identificar patrones de liderazgo en las jugadoras. Para ello elaboraron un listado de características frecuentemente asociadas a los líderes, lo sometieron a la evaluación de expertos en deporte y, posteriormente, a una prueba piloto con una muestra amplia. De este proceso extrajeron una lista definitiva de 19 ítems, a la que se suman 6 ítems de relleno para la presentación a las futbolistas. Se pidió que cada jugadora evaluara, en función del SLBI, a cada una de sus compañeras y también a sí misma. Además se pidió a los entrenadores que evaluaran a cada jugadora. Con esto se obtenían tres medidas de liderazgo, que luego se relacionaron con otras variables medidas. En la construcción del SLBI las propias autoras señalan sobre los resultados que "no tienen por qué ser necesariamente generalizables a otros grupos" y recomiendan que se extienda la investigación a "otros deportes, grupos de edad, grupos raciales y género" (Glenn y Horn, 1993, pág. 33). El SLBI fue posteriormente utilizado en una réplica de esta investigación, en la que se añadieron más variables de tipo psicosocial para determinar su relación con el liderazgo (Moran y Weiss, 2006).

Otros autores han aportado un nuevo sistema de evaluación del liderazgo del jugador, basándose en el ya referido modelo de tres dimensiones: social, de tarea y representación externa (Kogler-Hill, 2001). Formularon una serie de ítems sobre conductas referidas a cada una de las dimensiones (cuatro para cada una) y pidieron a los miembros del equipo que anotaran cuáles de sus compañeros habían realizado una, algunas o todas las conductas listadas. Se presentaron de forma separada cada una de las tres dimensiones. Si algún jugador era señalado por más de la mitad de sus compañeros en alguna de las tres evaluaciones se tomaría como líder que puntuaba alto en esa dimensión (Loughead, Hardy y Eys, 2006). En un siguiente estudio se amplió ligeramente el listado de ítems (se añadió uno por dimensión) pero se mantuvo el procedimiento (Eys, Loughead y Hardy, 2007).

Kozub y Pease (2001), en un estudio sobre la relación entre el liderazgo del entrenador y el del jugador en equipos de baloncesto de institutos estadounidenses, trabajaron también en la construcción de una escala de evaluación del liderazgo del jugador desarrollando el Player Leadership Scale (PLS). Para la construcción de la escala tomaron ítems del LSS y del Leader Behavior Description Questionnaire (LBDQ; Halpin y Winer, 1957) y los reformularon para hacerlos más apropiados de acuerdo al objetivo de la evaluación. La escala consta de 12 ítems, 6 por cada dimensión. En su aplicación se pidió a cada jugador que evaluara la capacidad de liderazgo de cada uno de sus compañeros en función de los ítems de la escala, de forma que luego se tomaron las puntuaciones otorgadas a cada jugador por sus compañeros, obteniendo como resultado que el líder tiende a puntuar alto en cada una de las dimensiones. Los ítems del PLS fueron utilizados en un estudio posterior con una muestra de estudiantes escolares ofreciendo resultados similares (Todd y Kent, 2004). A pesar de todo no existe información suficiente sobre la adaptación y/o validación del cuestionario 
para ser aplicado, ni se ofrecen características psicométricas para poder ser evaluado. Una ventaja de estas dos últimos métodos descritos, respecto investigaciones anteriores, es el desarrollo de una escala que sea plena y claramente consistente con un modelo teórico asumido. Particularmente, la PLS se fundamenta además en el modelo teórico predominante.

Con la intención de superar las limitaciones que se han señalado con respecto a las investigaciones precedentes, se ha desarrollado también la Escala de Evaluación de Liderazgo Deportivo (EELD). Esta escala ha sido elaborada como complemento del modelo conceptual integrador señalado al final de apartado teórico, a partir de investigaciones realizadas en España en equipos de fútbol y baloncesto de competición en categoría senior (Arce et al., 2008; Torrado, 2009). Para estas investigaciones se elaboró una escala inicial de 54 ítems referidos a conductas asociadas a las dimensiones previamente hipotetizadas en el modelo teórico, y que fueron definidas a partir de una profunda revisión bibliográfica previa además de aportaciones de los autores. A los participantes se les pedía que escogiesen al compañero que hubieran tenido, en ese momento o en el pasado, que considerasen que mejor había ejercido como líder de equipo. El objetivo era que estimaran la frecuencia con la que realizaba cada una de esas conductas que presentaba la escala.

A partir de estas valoraciones se realizaron análisis factorial exploratorio y confirmatorio de los datos, así como análisis de fiabilidad de los ítems y evaluación de consistencia interna de los factores. Se obtuvo una solución de una escala final de 30 ítems que respondía al modelo teórico asumido por los autores, de dos grandes dimensiones: orientación social y orientación de tarea, en el que mientras que el factor de orientación a la tarea (compuesta por 6 ítems) es unidimensional, el factor social se divide en cuatro subfactores, referidos a las dimensiones conceptuales empatía, influencia en la toma de decisiones, valores y empatía, (todas ellas conformadas también por 6 ítems cada una) que ofrecieron índices psicométricos satisfactorios (Arce et al, 2011; Torrado, 2009).

Al igual que en el caso de los modelos teóricos, la existencia de nuevas aportaciones viene a resolver ciertas limitaciones presentadas por las investigaciones previas, aunque deja abiertas posibles líneas para futuros trabajos. En el caso concreto de la EELD, en cuanto a que se presenta como la propuesta integradora que incluye las aportaciones principales de las anteriores escalas, si bien parece sostenerse sobre un consistente análisis estadístico será necesario obtener más datos, tanto para dotar de mayor consistencia a la estructura de dos grandes dimensiones que parece asentarse, como también para someter a contraste las dimensiones de segundo orden, en la misma línea que se apuntó con respecto al modelo teórico con el que se complementa. Además, resultaría conveniente trasladar las herramientas de evaluación a la aplicación en contextos reales, para comprobar la validez en cuanto al objetivo final de su utilidad práctica. Por último, con el fin de obtener un instrumento de medida válido para distintos contextos y poblaciones, sería conveniente que futuras investigaciones replicaran estos trabajos en poblaciones femeninas y en otros deportes colectivos.

\section{Conclusiones}

La investigación en el liderazgo deportivo se ha centrado mayoritariamente en el estudio del liderazgo formal del entrenador, que presenta en los trabajos de Chelladurai (1978, 1990; Chelladurai et al., 1988; Chelladurai y Saleh, 1978, 1980) una referencia fundamental tanto a nivel teórico, con el Modelo Multidimensional del Liderazgo, como metodológico, con el desarrollo de una escala de evaluación consistente con este modelo teórico, la LSS.

En el caso del liderazgo informal, aquel que desarrollan algunos jugadores entre sus iguales en el equipo, no existe todavía el mismo nivel de acuerdo sobre una referencia sólida ni en el aspecto teórico ni en los métodos de evaluación, si bien es posible señalar algunos avances que parecen consolidarse. Desde un punto de vista teórico las investigaciones llevadas a cabo proponen la idea de la definición del liderazgo ejercido por algunos jugadores en equipos deportivos en base a dos grandes dimensiones: orientación social y orientación a la tarea (Kim, 1992; Kozub y Pease, 2001; Rees, 1983; Rees y Segal, 1984; Todd y Kent, 2004). Investigaciones más recientes refuerzan este enfoque, ofreciendo una propuesta teórica integradora aportando una nueva visión más matizada y con más profundidad del modelo, específicamente en la orientación social (Arce et al., 2011; Torrado, 2009).

En cuanto al aspecto metodológico, la evaluación de este tipo de liderazgo ha sufrido ciertas variaciones en función del objetivo concreto del trabajo, mostrándose diferencias en función del sistema de evaluación empleado. Aunque se ha intentado adaptar algunos instrumentos provenientes de otro tipo de medidas, parece natural concebir que este liderazgo informal debe ser afrontado de manera específica también en los métodos de evaluación, y con instrumentos que se ajusten al modelo teórico predominante. De entre ellas destaca el PLS, si bien este trabajo se desarrolló en un contexto cultural y deportivo determinado con lo que se reduce su capacidad de generalización, además de presentar ciertas limitaciones metodológicas en su construcción.

Una propuesta reciente, la EELD, ofrece una aportación consistente con el modelo teórico establecido, además de aportar un análisis estadístico sobre la propia escala que supone un avance sobre investigaciones previas (Arce et al. 2011; Torrado, 2009).

En el futuro, las investigaciones deberían encaminarse a consolidar el modelo teórico de dos grandes dimensiones, social y de tarea, así como conocer más en profundidad la estructura de segundo orden subyacente. En el plano metodológico, y en consonancia con las apreciaciones teóricas, si bien la EELD se ha desarrollado en consistencia con la propuesta teórica predominante y sustentada en análisis estadísticos pro- 
fundos, es necesario someterla a nuevas investigaciones que consoliden una herramienta robusta que dote de consistencia al modelo señalado y contribuyan a comprobar su validez en un contexto de aplicación práctica, además de ampliar los trabajos a muestras femeninas y distintos deportes.

\section{Referencias}

1. Arce, C., Torrado, J., Andrade, E., Garrido, J. y de Francisco, C. (2008). Elaboración de una escala para la medida de la capacidad de liderazgo de los deportistas de equipo. Psicothema, 20, 913-917.

2. Arce, C., Torrado, J., Andrade, E. y Alzate, M. (2011). Evaluación del liderazgo informal en equipos deportivos. Revista Latinoamericana de Psicología, 43, 157-165.

3. Bales, R.F. (1953). The equilibrium problem in small groups. En T. Parsons, R.F. Bales, and E.A. Shils (Eds.), Working papers in the theory of action (pp. 111-161). New York: Macmillan.

4. Banzai, T. (1989). Leadership effects as a function of follower's personality characteristics. Japanese Journal of Education Psychology, 37, 107-116.

5. Bass, B.M. (1985). Leadership and performance beyond expectations. Nueva York: Free Press.

6. Bednarek, F., Benson, L. y Mustafa, H. (1976). Identifying peer leadership in small work groups. Small Group Research, 3, 307 -316. http://dx.doi. org/10.1177/104649647600700304

7. Caperchione, C., Mummery, W.K. y Duncan, M. (2011). Investigating the relationship between leader behaviours and group cohesion in within women's walking group. Journal of Science and Medicine in Sport, 14, 325-330. http://dx.doi.org/10.1016/j.jsams.2011.03.005

8. Carron, A.V., Hausenblas, H.A. y Eys, M.A. (2005). Group dynamics in sports ( $3^{a}$ ed.). Morgantown, WV: Fitness Information Technology.

9. Chelladurai, P. (1978). A contingency model of leadership in athletics. Tesis doctoral. Universidad de Waterloo. Canada.

10. Chelladurai, P. (1990). Leadership in sports: A review. International Journal of Sport Psychology, 21, 328-354.

11. Chelladurai, P., Imamura, H., Yamaguchi, Y., Oinuma, Y. y Miyauchi, T. (1988). Sport leadership in crossnational setting: the case of Japanese and canadian university athletes. Journal of Sport and Exercise Psychology, 10, 374-389.

12. Chelladurai, P. y Saleh, S.D. (1978). Preferred leadership in sports. Canadian Journal of Applied Sport Sciences, 3, 85-92.

13. Chelladurai, P. y Saleh, S.D. (1980). Dimensions of leader behaviour in sports: Development of a leadership scale. Journal of Sport Psychology, 2, 34-45.

14. Chen, B.D. (2006). The dynamics of leadership functions in a men's semi-professional basketball team in Taiwan, R.O.C. Tesis doctoral. Texas Woman's University.
15. Crespo, M., Balaguer, I. y Atienza, F. (1994). Análisis psicométrico de la versión española de la escala de liderazgo en el deporte de Chelladurai y Saleh en la versión entrenadores. Revista de Psicología Social Aplicada 4, 2-23.

16. Dupuis, M., Bloom, G.A. y Loughead, T.M. (2006). Team captains' perceptions of athlete leadership. Journal of Sport Behavior, 29, 60-78.

17. Eys, M.A., Loughead, T.M. y Hardy, J. (2007). Athlete leadership dispersion and satisfaction in interactive sport teams. Psychology of Sport and Exercise, 8, 281- 296. http://dx.doi.org/10.1016/j.psychsport.2006.04.005

18. Gill, D.L. y Perry, J.L. (1984). A case of study of leadership in women's intercollegiate softball. International Review of Sport Sociology, 14, 83-91. http://dx.doi. org/10.1177/101269027901400206

19. Glenn, S.D. y Horn, T.S. (1993). Psychological and personal predictors of leadership behavior in female soccer athletes. Journal of Applied Sport Psychology, 5, 17- 34. http:// dx.doi.org/10.1080/10413209308411302

20. Grusky, O. (1963). The effects of formal structure on managerial recruitment: a study of baseball organizations. Sociometry, 26, 345-353. http://dx.doi.org/10.2307/2786074

21. Halpin, A.W. y Winer, B.J. (1957). A factorial study of the leader behaviour descriptions. En R.M. Stodgill y A.E. Coons (Eds.), Leader behavior: Its description and measurement (pp. 39-51). Columbus, OH: Bureau of Bussiness Research, Ohio State University.

22. Hernández-Mendo, A. y Canto, J. M. (2003). El liderazgo en los grupos deportivos. En Hernández-Mendo, A. (Coord.), Psicología del deporte (vol. I): Fundamentos 2 (pp. 6-28). Buenos Aires: Tulio Guterman.

23. Holmes, R.M., McNeil, M., Adorna, P. y Procaccino, J. K. (2008). Collegiate student athletes' preferences and perceptions regarding peer relationships. Journal of Sport Behavior, 31, 338-351.

24. Homans, G.C. (1961). Social behavior: Its elementary forms. Nueva York: Harcourt, Brace and World.

25. Isberg, L. y Chelladurai, P. (1990). The Leadership Scale for Sports: its applicability to the Swedish context. Sweden: University College of Falun/Borlange.

26. Jacquart, P. y Antonakis, J. (2009). Does leader charisma predict presidential election outcomes? 14th Congreso Europeo de la EAWOP. Santiago de Compostela, mayo, 13-16.

27. Jowett, S. (2008). Coach-athlete relationships ignite sense of groupness. En M.R. Beauchamp y M. Eys (Eds.), Group Dynamics in Exercise and Sport Psychology (pp. 63-78). New York: Routledge.

28. Kim, M. (1992). Types of leadership and performance norms of school athletics teams. Perceptual and Motor Skills, 74, 803-806. http://dx.doi.org/10.2466/pms.1992.74.3.803

29. Klonsky, B. G. (1991). Leaders' characteristics in samesex sport groups: A study of interscholastic baseball and 
softball teams. Perceptual and Motor Skills, 72, 943-946. http://dx.doi.org/10.2466/pms.1991.72.3.943

30. Kogler-Hill, S.E. (2001). Team leadership. En P.G. Northouse (Ed.), Leadership: Theory and practice ( $2^{a}$ ed.) (pp. 161 - 187). Thousand Oaks, CA: Sage.

31. Kozub, S.A. y Pease, D.G. (2001). Coach and placer leadership in high school basketball. Journal of Sport Pedagogy: Teaching and Coaching in Sport, 7, 1-15.

32. Lacoste, P.L. y Laurencelles, L. (1989). The French validation of the Leadership Scale for Sports. Canadá: Universitè du Quèbec à Trois-Rivières.

33. Lee, M. J., Coburn, T. y Partridge, R. (1983). The influence of team structure in determining leadership function in association football. Journal of Sport Behavior, 6, 59-66.

34. LeUnes, A. (2008). Sport Psychology (4 $4^{\circ}$ ed.). Nueva York, NY: Psychology Press.

35. Liukkonen, J. y Salminen, S. (1989). Three coach-athlete relationship scales in relation to coaching climate. VI International Congress on Sport Psychology. Lathi, Finlandia. February 13-15.

36. Loughead, T.M. y Hardy, J. (2005). An examination of coach and peer leader behaviors in sport. Psychology of Sport and Exercise, 6, 303-312. http://dx.doi.org/10.1016/j. psychsport.2004.02.001

37. Loughead, T.M., Hardy, J. y Eys, M. (2006). The nature of athlete leadership. Journal of Sport Behavior, 29, 142-158.

38. Moran, M.M. y Weiss, M. R. (2006). Peer leadership in sport: Links with friendship, peer acceptance, psychological characteristics and athletic ability. Journal of Applied Sport Psychology, 18, 97-113. http://dx.doi. org/10.1080/10413200600653501

39. Mosher, M. (1979). The team captain. Volleyball Technical Journal, 4, 7-8.

40. Northouse, P.G. (2007). Leadership: Theory and practice (4 $4^{a}$ ed.). Thousand Oaks, CA: Sage.

41. Peus, C., Weisweiler, S. y Frey, D. (2009). Professor = leader? An investigation of faculty roles. 14th Congreso Europeo de la EAWOP. Santiago de Compostela, mayo, 13-16.

42. Rees, C. R. (1983). Instrumental and expressive leadership in team sports: A test of leadership role differentiation theory. Journal of Sport Behavior, 6, 17-27.

43. Rees, C. R. y Segal, M. W. (1984). Role differentiation in groups: The relationship between instrumental and expressive leadership. Small Group Behavior, 15, 109-123. http:// dx.doi.org/10.1177/104649648401500106
44. Serpa, S., Lacoste,P., Antunes, I., Pataco, V. y Santos, F. (1988). Methodology of translation and adaptation of an specific sport test: A Leadership Scale for Sports. II National Symposium of Psychology Research, Lisboa.

45. Todd, S. Y. y Kent, A. (2004). Perceptions of the role differentiation behaviors of ideal peer leaders: A study of adolescent athletes. International Sports Journal, 8, 105-118.

46. Torrado, J. (2009). Desarrollo de un modelo de medida del liderazgo en equipos deportivos. Tesis doctoral. Facultad de Psicología. Universidad de Santiago de Compostela.

47. Tropp, K. y Landers, D. (1979). Team interaction and the emergence of leadership and interpersonal attraction in field hockey. Journal of Sports Psychology, 1, 228-240.

48. Weiss, M. R., y Stuntz, C. P. (2004). A little friendly competition: Peer relationships and psychosocial development in youth sport and physical activity contexts. En M. R. Weiss (Ed.), Developmental sport and exercise psychology: A lifespan perspective (pp. 165 - 196). Morgantown, WV: Fitness Information Technology.

49. Wheelan, S.A. y Johnston, F. (1996). The role of informal members leaders in a system containing formal leaders. Small Group Research, 27, 33-55. http://dx.doi. org/10.1177/1046496496271002

50. Wright, A. y Côtè, J. (2003). A retrospective analysis of leadership development through sport. Sport Psychologist, 17, 268-291.

51. Yukelson, D., Weinberg, R., Richardson, P. y Jackson, A. (1983). Interpersonal attraction and leadership within collegiate sport teams. Journal of Sport Behavior, 6, 28-36.

52. Zaccaro, S.J., Rittman, A.L. y Marks, M.A. (2001). Team leadership. The Leadership Quartely, 12, 451-483. http://dx.doi.org/10.1016/S1048-9843(01)00093-5

53. Zhang, J., Jensen, B.E. y Mann, B.L. (1997). Modification and revision of the Leadership Scale for Sport. Journal of Sport Behavior, 20, 105-122.

Fecha de recepción: 11 de febrero de 2012

Fecha de recepción de la versión modificada: 9 de abril de 2012 Fecha de aceptación: 14 de mayo de 2012 